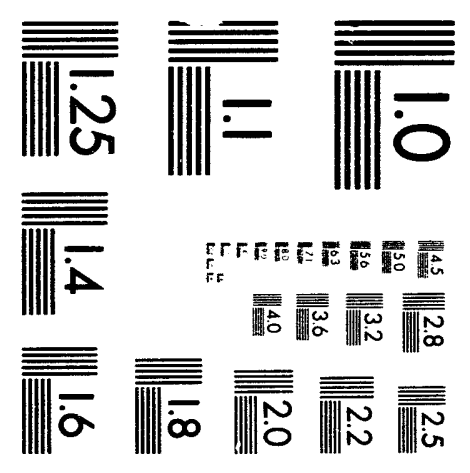



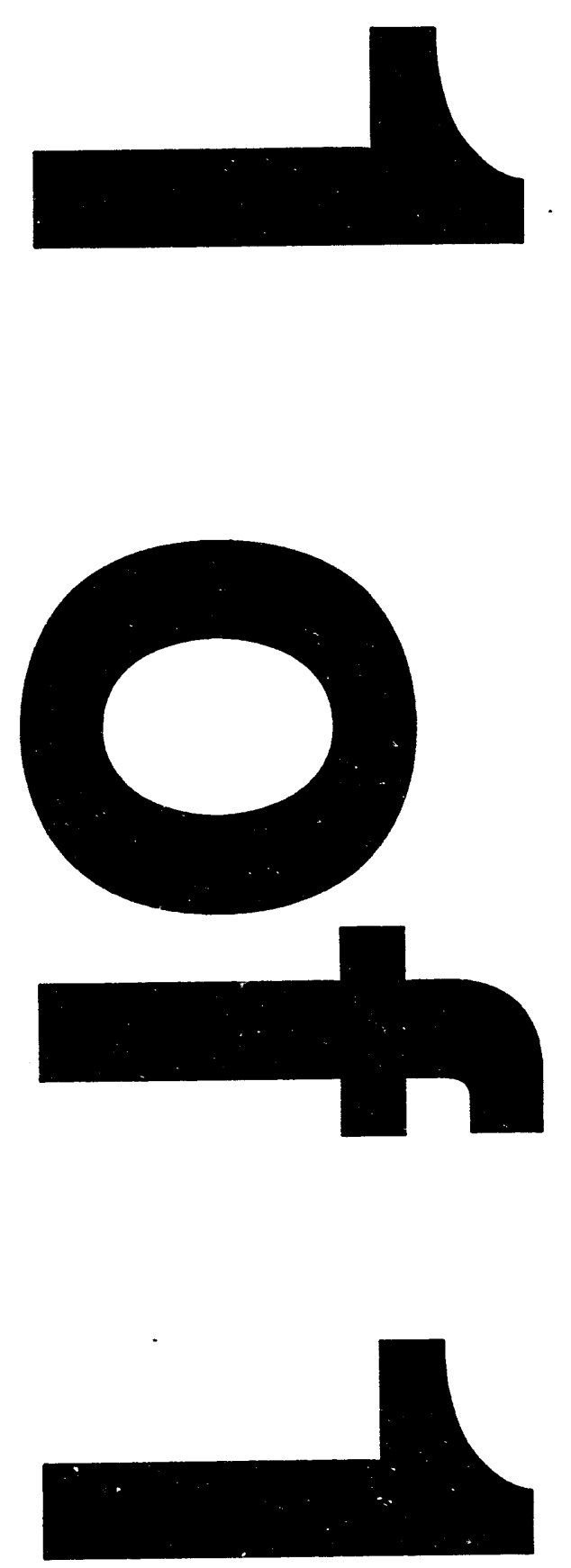
DOE/ER/54197-1

\title{
MARYLAND CONTROLLED FUSION RESEARCH PROGRAM
}

\author{
Progress Report \\ November 1, 1992 - October 31, 1993 \\ T. M. Antonsen, Jr., J. F. Drake, J. M. Finn, P. Guzdar, \\ A. Hassam, C. S. Liu, E. Ott \\ University of Maryland \\ College Park, MD 20742-3511
}

Amount of Unexpended Funds: \$0

May 1993

Prepared for the U.S. Department of Energy Under Grant Number DE-FG-02-ER54197

DISTRIBUTION OF THIS DOCUMENT IS UNLIMITED 
TASK I: Plasma Theory Principal Investigators: T. M. Antonsen, Jr.," J. F. Drake, ${ }^{* *}$ J. M. Finn, ${ }^{* * *}$ P. Guzdar, A. Hassam, ${ }^{*}$ C. S. Liu, ${ }^{*}$ E. Ott.* Additional Physicists: R. Kleva)

\section{TASK DESCRIPTION}

Purpose: The Maryland Theory Program is dedicated to the following tasks:

(i) To intensity our effort to develop an understanding of the fundamental processes which produce anomalous transport in tokamaks and to propose methods for enhancing confinement.

(ii) To. continue our investigation of tokamak edge plasma turbulence, sheared flow generation and the $\mathrm{L} / \mathrm{H}$ transition.

(iii) To continue our investigation of major disruptions with an emphasis on developing techniques for stabilization.

(iv) To continue collaborations with experimentalists and theorists of major fusion laboratories and university fusion centers for close interplay of theory, experiment, and simulation.

(v) To provide the theoretical support to the existing and proposed Maryland experimental programs in plasma diagnostics such as laser particle tagging and cyclotron radiation measurements from tokamaks.

(vi) To continue the education and training of scientists in plasma theory relevant to fusion.

\section{DISCLAIMER}

This report was prepared as an account of work sponsored by an agency of the United States Government. Neither the United States Government nor any agency thereof, nor any of their employees, makes any warranty, express or implied, or assumes any legal liability or responsibility for the accuracy, completeness, or usefulness of any information, apparatus, product, or process disclosed, or represents that its use would not infringe privately owned rights. Reference herein to any specific commercial product, process, or service by trade name, trademark, manufacturer, or otherwise does not necessarily constitute or imply its endorsement, recommendation, or favoring by the United States Government or any agency thereof. The views and opinions of authors expressed herein do not necessarily state or reflect those of the United States Government or any agency thereof.

*Professor

** Professor and contact person for this contract

${ }^{* * *}$ On leave to LANL 


\section{TECHNICAL PROGRESS}

In recent years, members of the Maryland Plasma Theory Group have made significant contributions to the national fusion theory program, and, in many cases, these theoretical developments helped to interpret experimental results and to design new experimental programs.

In the following, we summarize the technical progress in four major areas of tokamak research: (a) $\mathrm{L} / \mathrm{H}$ transition and edge turbulence and transport; (b) active control of micro-turbulence and transport; (c) major disruptions; and (d) the sawtooth crash.

\section{A. L/H Transition in Tokamaks}

The transition of the tokamak to a regime of improved confinement ( $\mathrm{L} / \mathrm{H}$ transition) has led to recognition of the importance of the edge plasma in controlling global confinement. Recent experimental measurements at UCLA and on DIII-D at G. A. have shown that poloidal rotation very likely plays an important role in the onset of the H-mode. Measurements on TEXT indicate that sheared rotation has a stabilizing influence on edge turbulence and locally reduces transport. Measurements at UCLA also point out the importance of poloidal asymmetry in understanding the $\mathrm{L} / \mathrm{H}$ transition.

During the past two years, we have developed a conceptual model of the tokamak edge in both L- and $\mathrm{H}$-mode. ${ }^{1,2}$ In this model we identify four important ingredients: poloidally asymmetric transport driven by the drift-resistive ballooning mode $;^{3,4}$ parallel flows of the order of the sound velocity $\left(c_{s}\right)$ produced by the asymmetric transport $;^{5,6}$ poloidal rotation (Stringer mechanism) produced by these parallel flows $;^{1,5}$ and finally order one $(\tilde{n} / n \sim 1)$ turbulence produced by the cross-field gradient of the parallel flows ( $V_{\|}^{\prime}$ instability). ${ }^{5,7}$ The nonlinear interaction between these four components determines whether the plasma remains in L-mode or makes a transition to the H-mode. When magnetic pumping is large, the poloidal rotation remains of the order of the diamagnetic level. Pcioidally asymmetric transport produces a significant variation of the density along flux surfaces in the edge and results in parallel flows of order $c_{s}$. Experimentalists on CCT, TEXT and DIII-D are presently attempting to confirm the presence of these parallel flows in $\mathrm{L}$ mode edge plasmas. When the magnetic pumping is weak, the Stringer mechanism (parallel flow) causes the edge plasma to spin, amplifying the existing diamagnetic level shear layer which is present in the L-mode. Poloidal rotation increases to $V_{\theta} \sim c_{g} a / q R$ when poloidal rotation competes with the parallel flow in smoothing the edge poloidal asymmetries. In the $\mathrm{H}$ phase the poloidal asymmetries and consequently parallel flows are greatly reduced. Transport is suppressed by sheared rotation, allowing steepened edge density gradients. 
In the following sections we describe the more detailed calculations we have completed and are presently pursuing to solidify the model presented above. This work was highlighted as an important new theoretical model of the $\mathrm{L} / \mathrm{H}$ transition in the 1992 IAEA summary talk by Dr. Pogutse. ${ }^{2}$ Two invited talks were presented at the 1992 APS meeting on this work.

\section{3D Fluid Simulation of the Nonlinear Drift Resistive Ballooning Modes in Tokamak Edge Plasmas}

The single most important experimental observation for identifying the cause of fluctuations and transport in tokamak edge plasmas is the strong inside-outside asymmetry of the anomalous particle flux (with a larger flux on the outside). This indicates that an instability with an outward ballooning character would be the most likely candidate for driving the observed particle flux. Since most edge plasmas are collisional, the only model which has the necessary requisites is the drift resistive ballooning mode (DRBM) ${ }^{3}$ Thus over the last two years we have studied the nonlinear behavior of DRBM turbulence, transport and the self-consistent generation of flows. ${ }^{4}$ This work first required a careful analytic analysis of the dominant scale lengths and time scales and second the development of a novel numerical algorithm for advancing the Braginskii fluid equations in a helical coordinate system based on a low order rational surface in the tokamak edge.

Our calculations have shown that:

i. In the nonlinear phase, these modes have a strong ballooning character. As a consequence, the turbulent transport caused by these modes has a strong poloidal asymmetry, i.e., peaked on the outside of the torus. Parallel flows from the outside to the inside of the torus develop as a result of the density asymmetry from the ballooning character of the transport.

ii. The magnitude of the anomalous transport $D^{a n}$ associated with these modes is $2 \times 10^{4} \mathrm{~cm}^{3} / \mathrm{sec}$ which is consistent with experimental observation.

iii. These modes can give rise to poloidal flows which are driven both by the Reynold's stress ${ }^{8-10}$ as well as the anomalous Stringer mechanism. The anomalous Stringer drive dominates at late time once parallel flows are well developed.

iv. The radial and poloidal correlation lengths were found to be in reasonable agreement with observations on TEXT. ${ }^{1}$

Thus the 3D simulations of the DRBMs have indicated that these modes have the potential for explaining edge fluctuations and transport in tokamaks. 


\section{Formation of the Shear Layer and the Development of Parallel Flows in Toroidal Edge Plasma}

Experimental measurements on TEXT and other tokamaks and stellarators indicates that a shear layer (region of sheared poloidal rotation) forms near the boundary between open and closed flux surfaces in the tokamak edge. This shear layer is present in Ohmic plasmas and the enhancement of this layer during the L/II transition leads to shear stabilization of edge turbulence and improved confinement in the H-mode. Thus, the development of a theoretical model of the shear layer is an important goal of the theory program.

The development of parallel and perpendicular flows in toroidal edge plasma has been investigated in the presence of an anomalous ballooning-like transport. ${ }^{6}$ The model is based on the Braginskii fluid-equations in an axisymmetric torus. A priority of this work has been to properly model the transition between open and closed field lines where the shear layer forms. Key results of this work are as follows:

i. The equilibrium density evolves so that the transport rate $D_{\perp} / L_{n}^{2}$ equals the sound rate, $c_{s} / \pi q R$. Since diffusion and sound rates are comparable, the variation of the density along a flux surface in the edge is of order unity and parallel flows of order $c_{s}$ are generally expected. This results also implies that confinement is linked to the parallel dynamics.

ii. In the open flux region the radial electric field is outward as a result of the Bohm sheath at the limiter or diverter plates. The poloidal rotation rate is at the diamagnetic level.

iii. When magnetic pumping is large the rotation in the closed flux region is equal in magnitude and opposite in direction to the ion diamagnetic drift (inward radial electric field), the usual neoclassical result.

iv. When magnetic pumping is weak, poloidal rotation in the closed flux region is amplified by the Stringer mechanism (with inward electric field) to a level close to the poloidal sound speed $\left(a c_{s} / q R\right)$.

\section{Linear Theory of Drift Resistive Ballooning Modes}

The conventional theor $y^{3}$ for the resistive ballooning modes relies on a two space scale analysis, which is justified for weak magnetic shear. ${ }^{12}$ We have first, analytically and numerically, studied the RMHD (one fluid) model for resistive ballooning modes with average favorable curvature in the weak shear limit. It is found the average curvature has a strong stabilizing influence and also leads to a localization of the mode. Increasing magnetic shear leads to destabilization of low mode numbers. High mode numbers are unaffected for $r q^{\prime} / q \leq 1$. We have next studied the Reduced 
Braginskii Two-Fluid model of the resistive ballooning modes both numerically and analytically. We find that with the inclusion of the average curvature affects the two scale analysis for weak shear and yields results which are significantly different from the conventional one fluid model. For $r q^{\prime} / q=1$, the low modes are more unstable, while the high mode numbers are unaffected.

\section{The $V_{\|}^{\prime}$ Instability in Tokamak Edge Plasma}

Our 3D simulations of DRBM have succeeded in explaining many aspects of experimental observations of edge turbulence as discussed earlier. However, one of the important features that is not explained is the large levels of density fluctuations observed in the edge. Experiments on TEXT indicate that $\delta n / n_{0} \sim 40 \%$. However, from sur 3D studies of the DRBM $\delta n / n \sim 10 \%$. Recently large-scale, axisymmetric vortices have been measured in the edge of CCT. These low mode number vortices can noi be curvature-driven at the low values of $\beta$ in these experiments. One important secondary instability which can enhance the fluctuations to the level observed and can drive long wavelength modes is the $v_{\|}^{\prime}$ instability. We have shown that ballooning transport in the edge leads to radially localized flows (scale length $\sim 2 \mathrm{~cm}$ ) of the order of $c_{y} .^{5,6}$ Such localized parallel flows are unstable to an electrostatic instability which transports momentum across $\mathbf{B}$, the $v_{\|}^{\prime}$ instability.

Two investigations of the $V_{\|}^{\prime}$ instability have been pursued. In the first, we explored the instability of a toroidal plasma to axisymmetric vortices driven by $v_{\|}^{\prime}$ to explain the recent observations on $\mathrm{CCT} .{ }^{13} \mathrm{We}$ demonstrated that axisymmetric vortices evolve to large amplitude if $\rho_{s} q R / a L_{n}>1$. In CCT because of the weak magnetic field this inequality is satisfied and such vortices are expected while in most other tokamaks the inequality is not satisfied and only nonaxisymmetric disturbances are unstable.

In the second investigation we have developed a fully nonlinear 3 dimensional code to study the nonlinear turbulence and transport of the $v_{\|}^{\prime}$ instability. ${ }^{7}$ We use a field aligned coordinate system with a sheared magnetic field. The equations that we evolve are the ion continuity equation and the parallel momentum equation. The perpendicular dynamics of the ions are due to the $E \times B$ drift and the polarization drift, and the electrons are presumed to respond adiabatically. We drive a background parallel velocity profile with radial gradients that mimic the flow pattern of the edge.

In dimensionless form, the equations contain three parameters, $\alpha=2 \pi q R \rho_{s} / L_{v}^{2}$ which is a ratio of the growth time to the parallel sound transit time, $\hat{\rho}=\rho_{s} / L_{v}$ which determines the strength of the polarization drift, and $\hat{s}=d \ln q / d \ln n$ which is a measure of the magnetic field shear. For the edge, $\alpha \gg 1, \hat{\rho} \ll 1$, and $\hat{s} \sim 1$. We examined the dependence of the turbulence and the transport on these parameters. Our most important result is that this instability drives large fluctuation levels $(30 \%$ $-50 \%$ ) and gives transport rates of the order $\sim \rho_{s} c_{s}$. The perpendicular scale 
length of the turbulence in both the $x$ and $y$ directions is of the order of the scale length of the velocity gradient. The parallel structure is determined by the parameter $\alpha$. As $\alpha$ increases, so does the number of wavelengths along the field, however, the perpendicular mode structure remains fairly unaffected. In agreement with this, the saturated transport rate also seems to be independent of $\alpha$. The value of $\rho$ has little effect on the saturated transport rate, however, it does play a critical role in initiating vortex mixing at the earlier nonlinear stages. The impact of magnetic shear is presently being explored. Preliminary indications are that magnetic shear is too weak to cause radial localization of the turbulence.

\section{B. Active Control of Micro-turbulence and Transport}

There is strong experimental evidence that perpendicular velocity shear may suppress micro-turbulence. In the past several years we have been in the forefront of research identifying mechanisms by which a plasma may be externally torqued to set up sufficient velocity shear to improve confinement. ${ }^{6,14-16}$ Our focus has been broad, examining ways tu drive rotation and suppress turbulence both in the edge and in the interior of the discharge. We have focused on two approaches to generate sheared flows: with neutral beams and with localized particle sources.

\section{Neutral Beam Driven Rotation}

We have suggested that NBI could be used to drive velocity shears. ${ }^{2,14-15}$ We have examined both poloidal injection and toroidal injection. In both cases, while the early calculations show that achievement of the above criterion will not be an easy matter, they also show that the proposed schemes are in the realm of feasibility. For poloidal injection, it may be possible to achieve a relatively narrow shear layer ( $\lesssim 20 \%$ of minor radius) with existing NBI injected poloidally. In toroidal injection, if the toroidal rotation speed can be made to exceed the sound speed, stabilization can be achieved.

We have actively been working with the DIII-D experimental group to plan an experiment on the poloidal injection of neutral beams.

\section{Spin-up from Axisymmetric Particle Fueling}

If a source of plasma is turned on in a tokamak, with a source rate larger on the outboard side of a flux surface than on the inboard side, a spontaneous poloidal spinup of the plasma can occur. We have recently demonstrated this ${ }^{16}$ in a calculation for an arbitrarily shaped, axisymmetric tokamak plasma where the particle (and momentum) sources may be nonaxisymmetric. Our calculation thus applies to particle 
sources arising from pellets, gas puffing, or centrally neutral beam fucled systems like TFTR supershots. The mechanism for the spin-up is the Stringer mechanism. The spin-up is destabilized provided the poloidally asymmetric portion of the particle fueling rate exceeds the damping rate of poloidal rotation from magnetic pumping reduced by a factor $\epsilon$, the inverse aspect ratio.

In the edge plasma the optimum location of the source for driving poloidal rotation is not on the outboard edge of the plasma but on either the top or bottom depending on the direction of the toroidal magnetic field. ${ }^{6}$ The optimum location is on the side towards which ions are drifting in the toroidal magnetic field. A source at this location enhances the naturally occurring Stringer-drive of poloidal rotation in the edge. To the extent that the diverter throat acts as a source of neutrals these results are consistent with the experimental observation that the power threshold for transition to the $\mathrm{H}$-mode is a minimum when the diverter throat is towards the direction of the ion drift. Exploration of the optimum location of particle sources for driving rotation is continuing.

This work was presented at the 1992 IAEA meeting. ${ }^{2}$

\section{Major Disruptions}

Major disruptions are an important limitation on the operating range of future large tokamaks such as ITER. The sudden loss of confinement during disruptions can cause severe damage to tokamaks. Therefore, it is important that disruptions are avoided during operation or, at least, that their damaging effects on the tokamak are ameliorated. We have previously simulated major disruptions in cylindrical geometry with a circular cross section using a reduced MHD Fourier code in which the reduced MHD variables are expanded in Fourier harmonics in the toroidal and poloidal angles. ${ }^{17}$ Key features of our simulations of major disruptions were confirmed in experiments on the TFTR tokamak. ${ }^{18}$ In particular, the initial loss of central confinement in the simulations is caused by the injection of a cold $m=1$ plasma bubble from the edge of the discharge to the center. Subsequently, experimental investigations of disruptions on TFTR have shown that the loss of confinement begins with the movement of a cold plasma bubble from the edge into the center, as predicted by our simulations.

In our recent work on disruptions we have continued to pursue an understanding of the underlying cause of disruptions by developing a new toroidal MIID code for exploring disruptions in a finite-aspect-ratio torus. At the same time we have explored some new techniques for suppressing disruptions by stabilizing the tearing mode. 


\section{Stabilization of the Tearing Mode by Overlap with Driven Alfvén Res- onant Islands}

The stability of the tearing mode in the presence of driven Alfven waves has been studied numerically. ${ }^{19}$ The model is reduced resistive MHD in a sheared slab with a driving electric field to produce a resistive equilibrium with a peaked current density. The traveling Alfvén waves are driven at a frequency such that the Alfvén resonance is near the region of large current gradient. The temporal evolution is studied as a function of the driving frequency and amplitude. The results indicate that the tearing mode can be completely stabilized. Stabilization occurs when the Alfvén resonance islands overlap the current layer, and is due to a combination of factors. The first is quasilinear flattening of the current profile. The second factor is that Alfvén waves at large amplitude in a sheared magnetic field have shear flow in their direction of propagation. This shear causes the tearing modes to propagate and saturate at a lower level. Therefore, at sufficiently large Alfven wave amplitude, the tearing mode is damped. The quasilinear flattening is due to self-consistent "magnetic field line" chaos, i.e., chaos in the characteristics of $d \mathbf{x} / d t= \pm \mathbf{v}_{A}$. These results suggest that it may be possible to stabilize tearing modes in a tokamak by exciting low frequency Alfvén waves of the same heiicity. Specifically, it may be possible to prevent disruptions (either density limit or current limit) by exciting $m=2, n=1$ Alfven waves at a frequency such that the inner Alfvén resonance is at the radius of maximum current gradient, which appears to be just inside the $q=2$ surface. Since the $q=2$ surface is near the edge, coupling to the kinetic Alfvén wave should not occur. It is envisioned that the Alfvén waves be launched in response to disruption precursors allowing the density and/or current to be decreased on a slower time scale. This work was given in an oral presentation at the 1993 Sherwood meeting.

\section{Toroidal MHD Code with Noncircular Cross Section}

We have recently written a fully nonlinear toroidal MHD code. In this code, the nonlinear equations for the magnetic field, momenta, thermal energy, and density are solved on a three-dimensional spatial grid. Spatial derivatives are evaluated to fourth order on the grid while time stepping in second order accurate with a leapfrog trapezoidal scheme. Grid oscillations resulting form convection are stabilized with a grid scale hyperviscosity. Since the code is fully explicit, it is particularly well suited for parallel processing. This fully gridded code is more suitable than Fourier codes for fully nonlinear three-dimensional simulations because a gridded code can more accurately resolve the steep gradients which arise nonlinearly. In Fourier codes, truncation of the spectrum gives rise to spurious ringing from steep gradients. We have tested our compressible MHD code on a model problem of the magnetic reconnection of two flux bundles. The results generated by our new compressible MHD code are essentially identical to those generated by our old reduced MHD code. 
We will now use our new toroidal MHD code to investigate the effect of toroidal aspect ratio and noncircularity on the limits to tokamak operating parameters posed by major disruptions. In particular, we will investigate the possibility that the operating range of tokamaks can be extended by varying the plasma shape and aspect ratio. We will also study the effectiveness of edge heating and/or current drive on controlling disruptions.

\section{Sawteeth in High Temperature Toroidal Plasmas}

The dynamics of the central region of high temperature tokamak plasmas is frequently dominated by the so-called sawtooth oscillations giving rise to a more or less periodic redistribution of the energy in the region. The trigger of these relaxations is the internal $n=1$ kink mode, where $n$ is the mode number for variation of quantities in the toroidal direction. This mode corresponds to a nearly rigid shift of the internal region of the plasma. Its suppression or control is considered to be important in present and future tokamaks. We have explored two important aspects of sawteeth. The first concerns the linear stability of the $n=1$ kink in the presence of trapped particles in a torus. The second concerns the rate of reconnection and the sawtooth crash time once the linear instability threshold is exceeded.

\section{Influence of Trapped Thermal Particles on the $n=1 \mathrm{Kink}$ in High Temperature Tokamak Plasma}

Energetic trapped particles are known to strongly modify the stability properties cf the $n=1$ internal kink mode in tokamaks. In high temperature discharges, similar interactions involving thermal particles can be shown to be important. ${ }^{20}$ When considering such interactions, it is essential to include the coupling to electrostatic perturbations that give rise to perturbed parallel electric fields. Thus, one is led to investigate the interaction between magnetic, MHD-like modes and electrostatic trapped particle modes. In order to study this interaction, we have modified the global stability code MARS to include the effects of trapped particles, both ions and electrons, and electrostatic perturbations. Using the code, we have investigated the stability of various profiles for circular and JET-shaped cross sections. The stability of the pressure-driven, ideal $n=1$ mode is strongly modified by the trapped particles. When the electrostatic potential is neglected, the trapped particles are generally stabilizing. However, with only one trapped species (electrons or ions), cases unstable in ideal MHD remain unstable and the mode acquires a significant rotation frequency. With both species of trapped particles treated by drift kinetic theory, the rotation is much reduced and the mode is stabilized well above the ideal-MHD threshold. If the electrostatic potential is retained, the mode structure can be strongly modified by the trapped particles via coupling of the $m>1$ side-bands to trapped particle modes, and the stabilizing effect is lost. Our results indicate that in high temperature tokamaks 
the linear stability on $n=1$ modes is dominated by trapped particle effects.

\section{Rapid Reconnection Driven by Electron Thermal Streaming in High Temperature Plasmas}

It is widely believed that the crash phase of the sawtooth is a consequence of the reconnection of magnetic field lines. In classical Sweet-Parker resistive MHD theory, the rate at which magnetic field lines reconnect is proportional to the square root of the Spitzer resistivity. However, the classical rate of reconnection based on Spitzer resistivity is far too small to account for the rapid phenomena observed during the crash of a sawtooth. For example, the time scale for classical magnetic field line reconnection based on the central temperature in the JET tokamak is more than ten times longer than the observed time scale for the drop in the central temperature during the sawtooth crash.

This apparent discrepancy between classical reconnection theory and experiment has motivated us to examine the effect of electron thermal streaming on the reconnection process. Electron thermal streaming can be included in the MHD equations by retaining the electron pressure in Ohm's law. We have carried out a series of reduced MHD simulations of the magnetic reconnection of two flux bundles using an Ohm's law which includes the parallel (to the magnetic field) electron pressure gradient. ${ }^{21}$ We have found that the thermal streaming of electrons along the magnetic field dramatically alters the structure of the dissipation region around the $\mathrm{X}$-point. Thermal streaming introduces a new physical scale length $\rho_{s}$ into the resistive MHD equations, where $\rho_{s}$ is the ion gyroradius based on the electron temperature. The dissipation region develops a two scale structure: a very small inner current layer and a somewhat larger flow layer in which the plasma flow is guided. Along the inflow direction to the X-point, the width of the extremely narrow current layer is proportional to the plasma resistivity $\eta$, while the outflowing plasma is guided in a broader channel of width $\rho_{s}$. In the outflow direction, the length of the current layer is given by $\rho_{s}$, while the length $L$ of the flow layer is macroscopic. As the reversed magnetic field lines are convected towards each other at the $\mathrm{X}$-point, they are compressed in a layer of width $\rho_{\text {s }}$ about the X-point. After reconnection, the tension in the compressed magnetic field lines is released accelerating the outflowing plasma to high speed. As a consequence, our simulations demonstrate that the inflow speed to the $X$-point is virtually independent of both $\eta$ and $\rho_{s}$. Thus, the surprising conclusion from our simulations is not only that the reconnection rate is enhanced in high temperature plasmas by electron thermal streaming, but also that the rate of reconnection remains large as both $\eta$ and $\rho$, become small. 


\section{PROPOSED RESEARCH}

\section{A. 3D Fluid Simulations of the Nonlinear Drift Resistive Ballooning Modes in Tokamak Edge Plasmas}

1. The primary focus of our 3D drift resistive ballooning mode (DRBM) simulations over the past year has been to identify the cause of fluctuations and transport in both $L$ and Ohmic discharges and to understand the mechanisms for the generation of poloidal rotation in toroidal plasmas. Our present goal is to include the necessary physics in our code to simulate the complete $\mathrm{L} / \mathrm{H}$ transition. Specifically, two important ingredients are being incorporated into our simulations: the scrape off layer (SOL) and magnetic pumping. In our $2 \mathrm{D}$ simulations of the generation of sheared flow, we have found that the inclusion of a SOL with appropriate particle sinks is essential to properly model the parallel flows and poloidal rotation which develops in the closed flux region. A simulation of the $\mathrm{L} / \mathrm{H}$ transition must therefore include the SOL and it has been included in our 3D DRBM code. The onset of the H-mode is controlled by the balance between sources and sinks of poloidal rotation. The dominant sink is magnetic pumping. In $\mathrm{L}$ mode or Ohmic plasmas magnetic pumping dominates the sources of poloidal rotation and neo-classical diamagnetic level flows in the closed flux region result in the usual sheared flow layers measured on TEXT. Magnetic pumping appropriate for a fully compressible plasma in the Pfirsch-Schlüter regime is being incorporated in our DRBM code. We will then proceed to complete simulations of the $\mathrm{L} / \mathrm{H}$ transition.

2. Our simulations demonstrate that although the Drift Resistive Ballooning Modes have many attractive features, they do not explain all features of the experimental observations of edge turbulence. One unexplained feature is the large level of the density fluctuations. Our 3D DRBM simulations give $\delta n / n_{0} \sim 10 \%$. Experimentally $\delta n / n_{0} \sim 0(1)$. The role of the $V_{\|}^{\prime}$ instability in enhancing turbulence levels in the edge region will be explored. We will continue to study the nonlinear development of turbulence by the $v_{\|}^{\prime}$ instability first in the simple slab with magnetic shear as discussed in the progress report and then on the complete torus with the DRBM turbulence.

3. Another direction that we hope to pursue is the inclusion of an electron temperature evolution equation in our $3 \mathrm{D}$ code. At present our code evolves the density, vorticity and the parallel momentum equations. Thus only the electrostatic drift resistive ballooning modes are studied. Data on TEXT shows that the electron temperature fluctuations are sizeable $\tilde{T}_{e} / T_{e} \sim .4 \tilde{n} / n_{0}$. Hence they cannot be neglected. The presence of the equilibrium electron temperature gradient can enhance the drive for the drift resistive ballooning modes - and drive temperature fluctuations. Thus the inclusion of the temperature equation is important. Also at present the heat flux is only due to loss of particles. With the temperature equation present the "off diagonal" transport becomes possible and the possibility of particle pinch caused by 
temperature fluctuations can be studied.

4. There is mounting evidence that there are two distinct regions in the tokamak separated by a sharp boundary. On TFTR this sharp region of separation is determined by the radial location to which the neutrals can penetrate. The presence of the neutrals can affect the collisionality of the electrons and ions. For the ions the collisions can enhance the inertia, whereas for the electrons the collisions with the neutrals can increase the resistivity. Preliminary linear stability studies of the effect of neutrals on the DRBM has shown that the lower poloidal mode numbers are destabilized whereas the high mode numbers are stabilized. We will include this additional physics in our 3-D nonlinear fluid simulations to determine the consequence on the nonlinear evolution. Also from the point of view of $\mathrm{L}-\mathrm{H}$ transitions the ion neutral collisions may play an important role in determining the threshold for the transition rather than the usual magnetic pumping, since for high ion collisionality (due to ion-ion collisions) the magnetic pumping decreases as $v_{i i}^{-1}$.

5. A fifth direction is the inclusion of the poloidal field or flux evolution. The ultimate goal of these studies is to provide an understanding of the $\mathrm{L} / \mathrm{H}$ transition in tokamaks. In D-IIID it is clear that since the edge plasma beta is larger than that in TEXT magnetic fluctuations are significant and hence it is necessary to include such effects. Also with the poloidal magnetic field evolution present the equations include the ideal ballooning instability which is dominated by magnetic fluctuations. Linear stability analysis of the D-IIID edge $\mathrm{H}$ mode plasmas indicate that the pressure profiles are within a factor of two of the ideal ballooning threshold. Thus the occurrence of ELM's (Edge Localized Modes) in the $\mathrm{H}$ mode phase could be due to the ideal ballooning modes. In fact using a simpler 2D Model with Rayleigh-Taylor instability (the analogue of the ideal ballooning instability) we have obtained interesting time dependent states resembling the signatures of ELM's. Thus by using experimental data as well as insights from our $2 \mathrm{D}$ studies we hope to be able to simulate the $\mathrm{L} / \mathrm{H}$ transition as well as the subsequent ELM phase using our $3 \mathrm{D}$ code.

\section{B. Active Control}

There are two key issues that will determine the relative success or failure of active control of plasma confinement by spinning the plasma. These are: (1) how "hard" is the $c_{s} / L$, threshold on $v_{\perp}^{\prime}$ required for stabilization of microinstabilities, and (2) what is the nature of collisionless magnetic pumping for large flow speeds.

To elaborate on (1), if significant suppression of turbulence is attained even before the $c_{s} / L$, threshold is reached, that would be advantageous and lower the power requirements to maintain the requisite $v_{\perp}^{\prime}$. We are investigating this question by examining numerically how saturated turbulence levels scale with increasing applied velocity shear. As far as (2) goes, it is well known that the magnetic pumping rate is nonlinear in the poloidal speed and decreases with increasing poloidal speed after 
a critical threshold. Such behavior affords the possibility of a "runaway" poloidal rotation after a critical applied force. We are investigating this area particularly as it applies to collisionless (banana) regimes. A related question we are investigating is the behavior of particle source induced Stringer spin-up in the banana regime.

\section{Major Disruptions in Finite-Aspect-Ratio, Noncircular Cross-Section Tokamaks}

An important conclusion of our simulations of density limit disruptions and the subsequent measurements on TFTR was that the state of the edge plasma plays a crucial role in the disruptive process. Specifically, when the edge temperature is sufficiently high $(\tilde{>} 50 \mathrm{eV})$ the instability of the plasma column must be characterized as an internal rather than an external kink and the nonlinear behavior of the instability does not. lead to a major disruption. By maintaining a very modest edge temperature major disruptions can theretore be suppressed and possibly completely eliminated.

A major caveat to these positive conclusions on major disruptions and their suppression in future large tokamaks concerns the impact of finite aspect ratio and shaping of the plasma cross-section on disruptions. The numerical simulations were based on the reduced resistive MHD equations in a periodic cylinder with circular crosssection. Because of their favorable stability properties to pressure driven modes, both ITER and future large tokamaks will very likely have a relatively low aspect ratio with a D-shaped cross section. It is well known that both finite aspect ratio and noncircular cross section can strongly impact the linear stability of the internal kink. How these two geometrical effects will influence the nonlinear development and injection of the cold bubble into the core plasma is unclear. Thus, hope that major disruptions may be suppressed in future tokamaks does not rest on a solid theoretical/computational foundation.

A 3D compressible MHD code to investigate major disruptions in finite aspect ratio tokamaks with noncircular cross sections is being developed. The basic algorithm for advancing the compressible 3D-MHD equations has been written and debugged. The toroidal modifications are now being incorporated into the equations. Self-consistent evolution of the plasma resistivity $\eta \sim T^{-3 / 2}$ will be included through a self-consistent temperature equation which will include both parallel and perpendicular conduction. The code is being built on a $3 \mathrm{D}$ toroidal grid $(R, Z, \phi)$ with a rectangular cross-section. Fourth-order spatial differencing has been combined with a second-order-accurate, leapfrog-trapezoidal time-stepping scheme. Improved numerical techniques will be explored and implemented when appropriate. The introduction of sources and sinks in the poloidal plane allows arbitrary specification of the shape of the outer flux surface and/or introduction of poloidal or toroidal limiters. Thus, the code will have maximum flexibility for studying MHD activity in a toroidal plasma. 
Once optimization and debugging of the code is complete, full scale simulations of density limit disruptions will be undertaken. Grids of order $400 \times 400 \times 40$ are presently envisioned with Lundquist numbers $S=\tau_{r} / \tau_{A}>10^{5}$, where $\tau_{r}=4 \pi a^{2} / \eta c^{2}$ is the resistive time and $\tau_{A}=R / c_{A}$ is the Alfvén time. A model radiation function will be incorporated into the temperature equation and the radiation rate will be ramped up to induce a contraction of the temperature and current profiles as observed in experiments. The impact of the poloidal plasma shape and aspect ratio on the growth of the $m=n=1$ kink and injection of the bubble into the central plasma will be explored. Of particular interest is also the role of the edge plasma in controlling the character of the kink - whether it is an internal kink, which does not lead to a major disruption or an external kink, which does. The important question is whether the maintenance of a modest edge temperature in a large D-shaped tokamak can prevent the formation of the external kink and therefore inhibit or prevent major disruptions.

\section{Sawteeth - Kinetic Theory of $n=1$ Instabilities}

The dynamics of the central region of high temperature tokamak plasmas is frequently Jominated by the so-called sawtooth oscillations giving rise to a more or less periodic redistribution of the energy and magnetic flux in the region. The trigger of these relaxations is the internal $n=1$ kink mode, where $n$ is the mode number for variation of quantities in the toroidal direction. This mode corresponds to a nearly rigid shift of the internal region of the plasma. Its suppression or control is considered to be important in present and future tokamaks.

In its ideal and most robust form, the mode grows on a time scale over which the plasma is ideally conducting. We have recently shown that this quasi-ideal $n=1$ mode is strongly modified by the presence of trapper thermal particles. A similar modification can be expected to occur for the non-ideal reconnecting $n=1$ mode in which the magnetic topology is changed. We thus propose to continue our investigations of the effect of trapped particles on $n=1$ modes by incorporating a model for reconnection into the global stability code MARS-K. This code was originally developed to investigate the ideal and resistive stability of tokamak plasmas in the MHD limit. We have recently modified the code (in collaboration with $\mathrm{A}$. Bondeson of the Centre de Recherches en Physique des Plasmas in Lausanne Switzerland) to treat the trapped thermal particles kinetically. We intend to continue this collaboration by developing an appropriate model for the reconnecting layer at each rational surface. 


\section{REFERENCES}

1. A. B. Hassam, T. M. Antonsen, Jr., J. F. Drake and C. S. Liu, Phys. Rev. Lett. 66, 309 (1991).

2. J. F. Drake, T. M. Antonsen, J. M. Finn, P. N. Guzdar, A. B. Hassam, C. S. Liu, D. McCarthy and F. W. Waelbroeck, in Plasma Physics and Controlled Nucl. Fusion Res. (IAEA, 1992).

3. D. McCarthy, P. Guzdar, J. F. Drake, T. M. Antonsen and A. B. Hassam, Phys. Fluids B 4, 1846 (1992).

4. P. N. Guzdar, J. F. Drake, A. B. Hassam, D. McCarthy and C. S. Liu, Proceedings of the First Energy Research Power Super Computer Users Symposium, May 21-22, 1991, Gaithersburg, MD (published by US DOE) article 15 and UMLPF \#92-023; P. Guzdar, J. F. Drake, A. B. Hassam and C. S. Liu, submitted to Phys. Fluids B (1992).

5. J. F. Drake, A. B. Hassam, P. N. Guzdar, C. S. Liu, and D. M. McCarthy, Nucl. Fusion Lett. 32, 1657 (1992).

6. D. R. McCarthy, J. F. Drake, P. N. Guzdar and A. B. Hassam, Phys. Fluids B5, 1188 (1993).

7. D. R. McCarthy, J. F. Drake and P. N. Guzdar, International Sherwood Theory Conference 1993 (Newport, RI), paper 3C26.

8. P. H. Diamond and Y. B. Kim, Phys. Fluids B 3, 1626 (1991).

9. J. Drake, J. Finn, P. Guzdar, V. Shapiro, V. Shevchenko, F. Waelbroeck, A. Hassam, C. S. Liu, and R. Sagdeev, Phys. Fluids B Lett. 4, 488 (1992).

10. J. Finn, J. Drake, and P. Guzdar, Phys. Fluids B4 2758 (1992).

11. A. Wooton et al., Phys. Fluids. B 2, 2879 (1990).

12. S. Novakovskii, P. N. Guzdar, J. F. Drake, C. S. Liu, and F. N. Waelbroeck, International Sherwood Theory Conference 1993, paper $1 \mathrm{C} 14$.

13. D. R. McCarthy, J. F. Drake and P. N. Guzdar, Phys. Fluids B (in press).

14. A. B. Hassam, Comments on Plasma Phys. and Controlled Fusion 14, 275 (1991).

15. A. B. Hassam and F. L. Waelbroeck, Proceedings of Workshop on New Ideas in Tokamak Confinement, La Jolla, CA (1992).

16. A. B. Hassam and T. M. Antonsen, submitted to Phys. Fluids B.

17. R. G. Kleva and J. F. Drake, Phys. Fluids B 3, 372 (1991). 
18. E. D. Frederickson, K. McGuire, J. F. Drake, R. G. Kleva, and the TFTR MHD Group, Nucl. Fusion (in press).

19. J. M. Finn, International Sherwood Theory Conf. 1993, paper xxx.

20. T. M. Antonsen and A. Bondeson, International Fusion Theory Conf. 1993, paper $2 \mathrm{C} 6$.

21. R. G. Kleva, J. F. Drake, and F. W. Waelbroeck, submitted to Phys. Rev. Lett. 1992. 


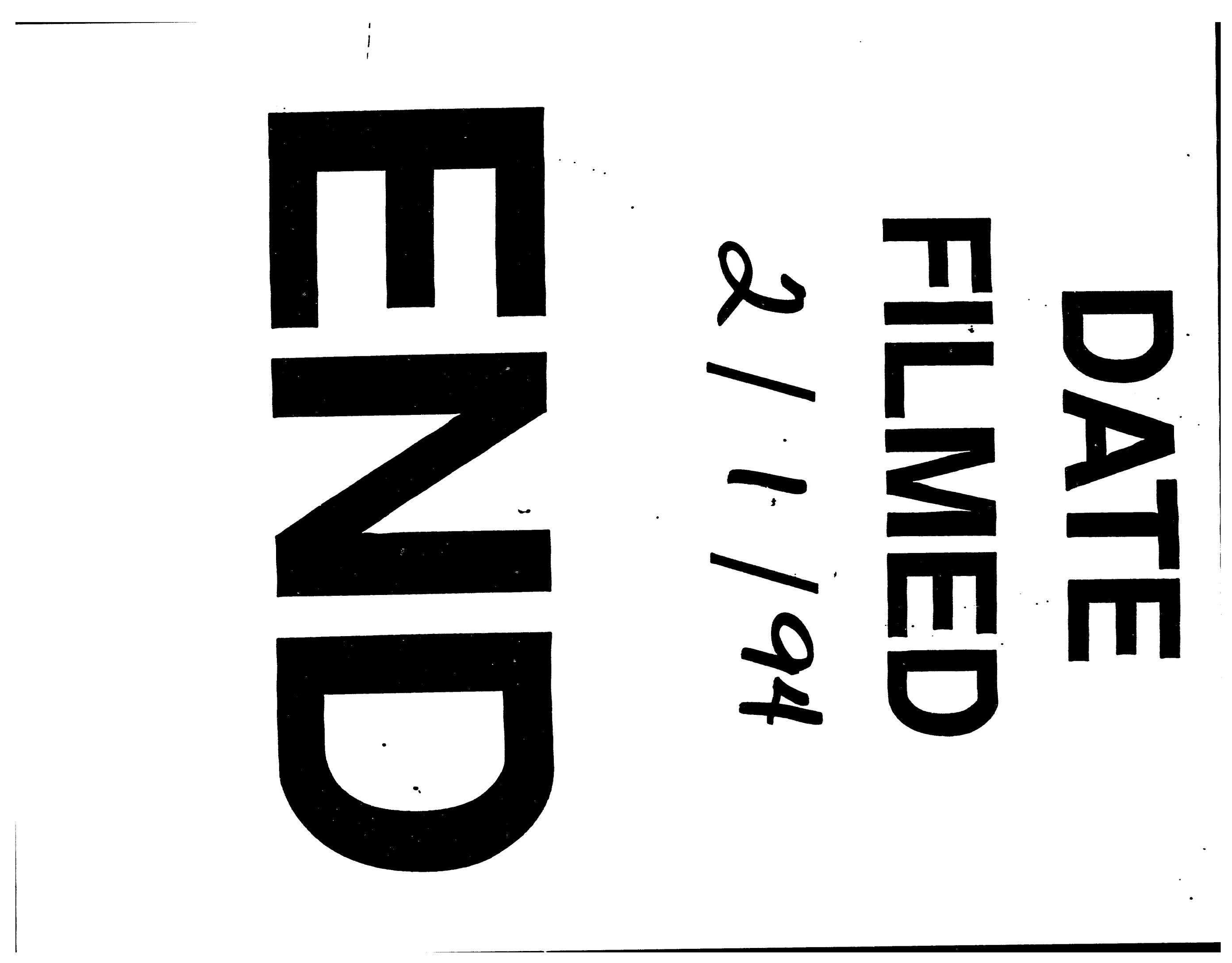




$$
\overline{\bar{s}}
$$

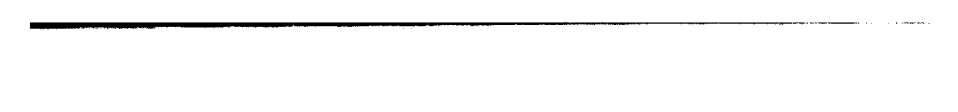
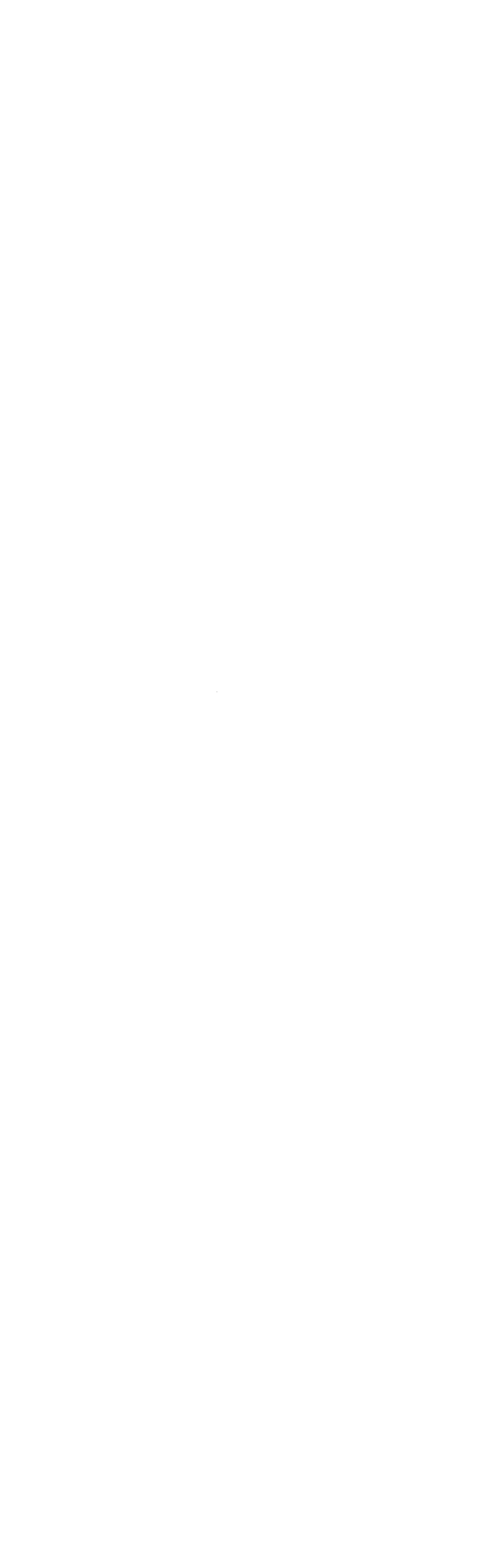